\title{
Entraining and copying of temporal correlations in dissociated cultured neurons
}

\author{
Terri Roberts ${ }^{1}$, Kevin Staras ${ }^{1}$, Philip Husbands ${ }^{2}$, Andrew Philippides ${ }^{2}$ * \\ ${ }^{1}$ School of Life Sciences, University of Sussex, Brighton, UK \\ \{T.P.Roberts,K.Staras\} @sussex.ac.uk \\ ${ }^{2}$ Centre for Computational Neuroscience and Robotics, Department of Informatics, \\ University of Sussex, Brighton, UK \\ \{andrewop, philh\}@sussex.ac.uk
}

\begin{abstract}
Here we used multi-electrode array technology to examine the encoding of temporal information in dissociated hippocampal networks. We demonstrate that two connected populations of neurons can be trained to encode a defined time interval, and this memory trace persists for several hours. We also investigate whether the spontaneous firing activity of a trained network, can act as a template for copying the encoded time interval to a naive network. Such findings are of general significance for understanding fundamental principles of information storage and replication
\end{abstract}

Keywords: Temporally correlated activity, Microelectrode array, neural network, dissociated cultured neurons

There is substantial current interest in understanding the way that temporal information is encoded in neural networks but the underlying mechanisms remain poorly defined. One emerging idea is that the temporal architecture of a circuit may be readily shaped by the timing of the input that the network receives [1]. If this is the case, how temporal correlations can be both entrained within, and copied between, networks of neurons could be significant with regards to the transfer of information between neural circuits. Here we set out to investigate whether such entraining and copying is possible in dissociated hippocampal networks.

To do this, we exploited multi-electrode array (MEA) technology. MEAs are a micro-engineered grid of extracellular electrodes providing a robust solution for sampling network spiking activity - effectively 'nodes' representing collective output of one or several neurons local to an electrode - as well as allowing the application of targeted stimulation. We have developed a method that allows us to grow neuronal cultures from dissociated hippocampal neurons which form mature highly interconnected networks in 10-12 days in vitro on which we can effectively record and impose activity patterns (Fig. 1 a-c). Using an MEA also demonstrates the possibility of being able to entrain temporal correlations in neural networks using a biohybrid device. 
a

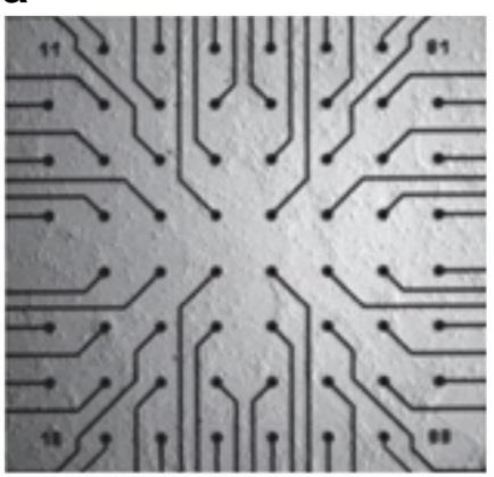

C
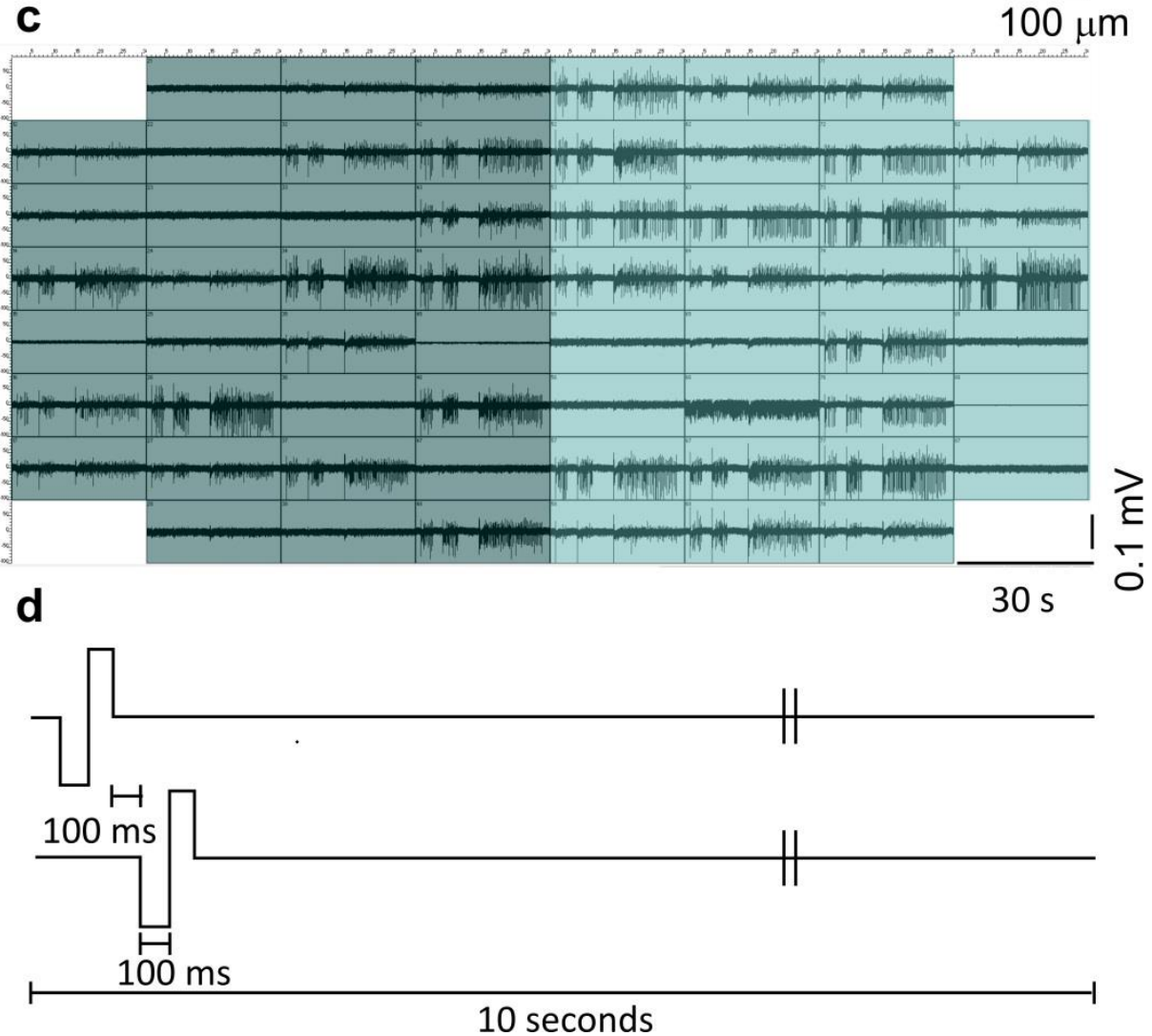

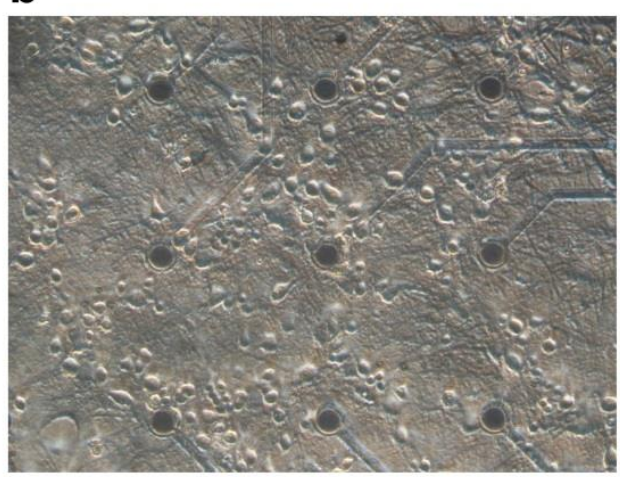

$\overline{100 \mu \mathrm{m}}$
10 seconds

Fig. 1. Typical appearance and output of hippocampal cultured neurons on multi-electrode array (MEA). (a) Image of 64 electrode MEA. (b) High-density neuronal cultures ( 14 DIV) used for experiments. (c) Typical readout of activity in 64 electrode recording. A nodes are shaded in dark, B nodes shaded in light. (d) Training protocol. Schematic shows timing of stimulation for A nodes (top) and B nodes (bottom) 


\section{a Spontaneous}

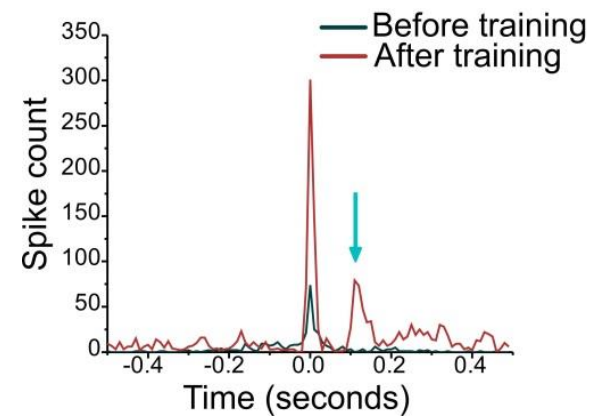

b

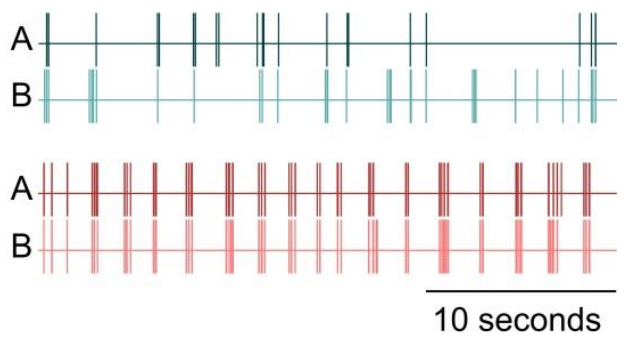

Fig. 2. Neural activity before and after training. (a) Cross-correlogram (A->B) before (green) and after (red) training for an example experiment. Training results in a robust peak at $\sim 120 \mathrm{~ms}$ (arrow). (b) Sample of node activity before (blue) and after (red) training showing training leads to synchronized activity. Spikes are seen as vertical lines in the subplots to the right.

We used this preparation to test whether it is possible to train two connected populations of neurons to encode a defined time interval. To do this, we initially divide the population of nodes into two sub-groups, 'A' and 'B' (Fig. 1c). The training paradigm (Fig. 1d) consists of electrical stimulation imposed onto A nodes followed, at a set time-interval $(100 \mathrm{~ms})$, by stimulation applied to B nodes and repeated 360 times over $1 \mathrm{~h}$. Using this paradigm, a defined time interval can be readily encoded in the firing relationship of the neural sub-populations stimulated (Fig. 2). In further experiments, we have seen that the encoded change can be persistent for several hours and that the fidelity of encoding changes when the training time interval is modulated.

In a second type of experiment, we are starting to explore the replicative capabilities of the stored information. Specifically, the objective is to use the post-training spontaneous activity recorded in $\mathrm{A}$ and $\mathrm{B}$ (which encodes the entrained $\mathrm{A}->\mathrm{B}$ temporal relationship) as the basis for a training paradigm imposed on a new 'naive' neuronal culture. Our preliminary experiments indicate that a trained network can indeed act as a template for replicating the same timing relationship in a new network. To date we have found that the effectiveness of this training paradigm is substantially lower than the original A->B training. This may relate to the choice of trained data sequences used as the basis for the secondary training. We are currently optimizing the training length and other parameters to improve the fidelity of the copying.

Here we exploit MEA technology to explore features of temporal encoding in hippocampal neural networks. We show robust storage of a defined time interval between the activity of two neuronal populations that becomes a feature of their spontaneous firing patterns after training. Such findings have broad similarities to those reported using large cortical networks in brain slice [1] but our results suggest that, remarkably, this capability is preserved in small reconstituted circuits. Moreover, initial results support the idea that, under appropriate conditions, this temporal relationship can 
act as a template for replication in independent networks. As such, this information replication mechanism offers a potential substrate on which complex copying properties - for example Darwinian neurodynamics [2] - may operate. This work is thus a step towards the empirical verification of the Neuronal Replicator Hypothesis (NRH) that states that a Darwinian process of production of adaptations by natural selection can run in real-time in the neuronal networks of the brain during its lifetime $[2,3,4]$.

Indeed, this work is motivated by a desire to better understand the dynamics of information transfer between neural circuits and in particular to examine the possibility of neural replicator dynamics in biological neuronal networks [3]. As well as being of benefit to neuroscience, an understanding of such dynamics points the way towards new kinds of biomorphic architectures for controlling adaptive behavior [5,6]. For instance, biomorphic neural devices and brain-machine interfaces could be designed to exploit the kinds of replicator dynamics uncovered in these studies. The use of an MEA to entrain information within a real neuronal network also raises the possibility of hybrid adaptive systems that incorporate cultures as dynamic information processing elements. However, the current work is in its infancy and much more fundamental science is needed to understand, let alone exploit, such information transfer.

Acknowledgements. This project has received funding from the European Union's Seventh Framework Programme for research, technological development and demonstration under grant agreement no 308943

\section{References}

1. Johnson, H.A., Goel, A., Buonomano, D.V.: Neural Dynamics of In Vitro Cortical Networks Reflects Experienced Temporal Patterns. Nat. Neurosci. 13, 917-919 (2010)

2. Fernando, C., Goldstein, R., Szathmáry, E.: The neuronal replicator hypothesis. Neural Comput. 22, 2809-2857 (2010)

3. Fernando, C., Karishma, K.K., Szathmáry, E.: Copying and Evolution of Neuronal Topology. PLoS ONE, 3, e3775 (2008)

4. Fernando, C., Szathmáry, E., Husbands, P.: Selectionist and Evolutionary Approaches to Brain Function: A Critical Appraisal, Front. Comput. Neurosci. 6, 24 (2012)

5. Fernando, C., Szathmáry, E.: Chemical, Neuronal and Linguistic Replicators. Towards an Extended Evolutionary Synthesis. In Pigliucci, M., Müller, G. (eds) Evolution: The Extended Synthesis, Cambridge, MA, MIT Press, 209-249 (2010)

6. Shim, Y., Husbands, P.: Incremental Embodied Chaotic Exploration of Self-Organised Motor Behaviours with Proprioceptor Adaptation. Front. Robot. AI, 2,7 (2015) 